\title{
Effect of turning-Q laser in combination with topical Chuangfukang collagen mask to improve facial appearance, greenish brown birthmark area and color depth of patients with facial greenish brown birthmark
}

\author{
Chu-na ZHENG ${ }^{1+}$, Peiping $\mathrm{WANG}^{2+}$, Dongying $\mathrm{YAO}^{3}$, Xiaojuan $\mathrm{FANG}^{4}$, Jianglin $\mathrm{WU}^{5}$, Xiaoqing ZHENG ${ }^{6 *}$, \\ Changmin $\mathrm{KE}^{7 \star}$ (1)
}

\begin{abstract}
To explore the effect of turning-Q laser in combination with topical Chuangfukang collagen mask to improve facial appearance, greenish brown birthmark area and color depth of patients with facial greenish brown birthmark. 130 female patient were selected as the study object. The patients were treated with turning-Q laser treatment instrument $1064 \mathrm{~nm}$, while the control group was treated with water bags for 20 minutes after cold irradiation. In the control group, the cure rate and total effective rate were 52.31\% (34/65) and 84.62\% (55/65), respectively, which were 67.69\% (44/65) and 95.38\% (62/65) (all P < 0.05). In the first week after treatment, the reduction rate (more than $80 \%$ ) of the area of facial greenish brown birthmark in the experimental group was significantly more than that in the control group $(\mathrm{P}<0.05)$. In six months after the treatment, only one case of dry desquamation occurred in the experimental group; three cases of dry desquamation, three cases of pruritus and two cases of rubella occurred in the control group. The application of turning- $Q$ laser in combination with topical Chuangfukang collagen mask in the treatment of facial greenish brown birthmark can significantly improve the clinical efficacy and the facial appearance.
\end{abstract}

Keywords: greenish brown birthmark; turning-Q laser; Chuangfukang collagen mask; pigmentation; curative effect.

Practical Application: The treatment of facial greenish brown birthmark.

\section{Introduction}

Facial greenish brown birthmark, also known as zygomatic mother spot and acquired bilateral nevoid spot of Ota, is a congenital non hereditary skin pigmentation disease, mainly distributed symmetrically on both sides of zygoma, involving forehead, temporal, periocular and alar nose, pigmentation on the dermis, showing round or oval cyan or light brown patches, with clear edge and mutual fusion of adjacent lesions (Hui et al., 2017). Over time, the color of greenish brown birthmark deepens gradually and the scope continues to expand. Although there are generally no self-conscious symptoms, it is easy for female patients to have self abasement due to its great influence on the external beauty of face, especially for young and middle-aged women with high aesthetic requirements for appearance, their physical and mental health may also be affected, and they are eager to be treated (Maligieri et al., 2017). In the past, facial greenish brown birthmark is usually treated through grinding and chemical peeling, accompanied with pigmentation and scar and etc. risk of complications (Chathra \& Mysore, 2018). With the rapid development of medical science and technology in recent years,, cosmetic medical technology has also made progress. Laser technology has replaced the traditional treatment method and become a safe and effective clinical treatment method (Imrigha et al., 2017). This study is to explore the effect of turning-Q laser in combination with topical Chuangfukang collagen mask to improve facial appearance, greenish brown birthmark area and color depth of patients with facial greenish brown birthmark. The specific contents are as following.

\section{Materials and methods}

\subsection{General data}

A total of 130 female patients with facial greenish brown birthmark from June 2017 to August 2018 were selected as the study object. According to treatment schedule, the patients were divided into control group (65 cases) and experimental group (65 cases). The average age of patients in the groups was 16-52 years old and the course of disease ranged from 1 to 10 years. The average age of the control group was $(35.68 \pm 4.32)$ years, and the average course of disease was $(5.43 \pm 1.25)$ years. The location of skin: bilateral zygomatic: 28 cases $(43.08 \%)$, 
frontotemporal zygomatic: 17 cases (26.15\%), temporal zygomatic: 13 cases $(20.00 \%)$, frontotemporal zygomatic and periocular: 7 cases $(10.77 \%)$; the average age of the experimental group was $(36.02 \pm 4.41)$ years, and the average course of disease was $(5.36 \pm 1.22)$ years. The location of skin: bilateral zygomatic: 27 cases (41.54\%), frontotemporal zygomatic: 18 cases $(27.69 \%)$, temporal zygomatic: 12 cases (18.46\%), frontotemporal zygomatic and periocular: 8 cases $(12.31 \%)$; All of them were round or oval color spots with symmetrical distribution and clear edge, and the forehead and periocular parts were distributed in sheet shape. Those who had skin cancer, mental disease and were treated with freckle removing drugs within one month before admission were excluded. There was no significant difference between the two groups $(\mathrm{P}>0.05)$. The study was approved by the ethics committee.

\subsection{Research method}

Before treatment, all patients will clean face cosmetics and oil, take photos of the face for archiving, and use them for comparison of the therapeutic effect before and after treatment; all patients should be treated with turning-Q laser treatment instrument $1064 \mathrm{~nm}$. The instrument is turning-Q laser needle (wavelength $1064 \mathrm{~nm}$ ) produced by HEFEI GUANGAN KEJI KAIFA CO.LTD Before laser irradiation, 5\% compound lidocaine cream will be evenly applied in the treatment area and sealed with preservative film for one hour; laser parameter setting: $9.0-12.0 \mathrm{~J} / \mathrm{cm}^{2}$, spot diameter of $2 \mathrm{~mm}$, pulse width of $100 \mathrm{~ns}$, frequency of 1.0 or $2.0 \mathrm{~Hz}$, and parameters should be adjusted reasonably according to the actual situation of patients' age, skin color, skin texture, etc.; the operator holds the handle of laser needle perpendicular to the treatment site and scans the skin lesions one by one, After irradiation, the skin color was deepened and the surface turned gray. The interval between two laser treatments was 3-6 months, and the treatment times were 2-6 times.

Control Group Upon laser irradiation, the patients in control group were treated with cold compress in water bag for 20 minutes once a day for 5 days; treated with antibiotic ointment for 7 days; do not touch water within 7 days after laser treatment and prevent from sun exposure within 4-6 weeks.

Experimental Group The patients in experimental group were treated with laser irradiation immediately after cold application. Chuangfukang collagen mask 20min (mask was pre-refrigerated), pressed lightly on the edge of the mask so that it adhered to the face of the patient, once a day, the mask was applied for 5 days on a continuous cold basis. Upon laser treatment, the antibiotic ointment was applied every day for 7 days; do not touch water within 7 days after laser treatment and prevent from sun exposure within 4-6 weeks.

\subsection{Evaluation of curative effect}

Observation index: one week after treatment, the patients' faces were photographed and archived, and compared with the photos before treatment. The reduction rate of the area of facial greenish brown birthmark in the two groups was less than $40 \%$, $40 \%-60 \%, 60 \%-80 \%, 80 \%$ and the incidence of pigmentation were counted respectively. Six months after the treatment, the dry and desquamation rate, pruritus rate and erythema rate and etc. adverse reaction rate of the two groups were counted and compared.

Determination of curative effect (Simunovic et al., 2017): six months after treatment, the patients' faces were photographed again for archiving. Compared with the photos before treatment, the improvement of the patients' facial appearance was observed. $91 \%-100 \%$ of the skin color disappeared and no scar was cured; $61 \%-90 \%$ of the skin color obviously became lighter and no scar was effective; $31 \%-60 \%$ of the skin color became lighter and no scar was improved; $30 \%$ or less of the skin color became lighter and no effect. The cure rate and the total effective rate were compared. Total effective rate $=($ cured cases + effective cases $) /$ total cases $\times 100 \%$.

\subsection{Statistical analysis}

The data of patients and research results are processed by SPSS 20.0 software. The measurement data are expressed as mean \pm standard deviation $(\mathrm{x} \pm \mathrm{s})$, $\mathrm{t}$-test between groups, percentage (\%), chi-square test between groups and rank and $\mathrm{Z}$ test between groups. If $\mathrm{P}<0.05$, the difference is statistically significant.

\section{Results}

\subsection{Comparison of cure rate and total effective treatment rate between the two groups}

The cure rate and total effective treatment rate are obviously higher than that of control group, and the difference between the two groups is statistically significant $(P<0.05)$. Refer the data in Table 1.

\subsection{Comparison of damaged skin area and pigmentation rate of the two groups}

In the first week after treatment, the reduction rate (more than $80 \%$ ) of the area of facial greenish brown birthmark in the experimental group obviously higher that that of control group, and the pigmentation rate was lower than that in the control group $(\mathrm{P}<0.05)$. Refer data in Table 2 and Figure 1.

\subsection{Comparison of incidence of adverse reactions of the two groups}

Six months after treatment, only one case of dry desquamation occurred in the experimental group; three cases of dry desquamation, three cases of pruritus and two cases of rubella occurred in the control group, the difference between the two groups was statistically significant $(\mathrm{P}<0.05)$ (Table 3$)$.

\section{Discussion}

Greenish brown birthmark are especially common in young and middle-aged women, especially in women after childbirth. The specific causes of the disease have not been fully identified, which may be related to genetic factors, environmental factors, ultraviolet rays and long-term make-up (Zhang et al., 2019). Some studies have suggested that the main reason for formation of 
Table 1. Comparison of Cure Rate and Total Effective Treatment Rate between the Two Groups [n (\%)].

\begin{tabular}{|c|c|c|c|c|c|c|}
\hline Group & Cases & Cured & Effective & Improved & Invalid & Total Effective Rate \\
\hline Control group & 65 & $34(52.31)$ & $21(32.31)$ & $9(13.85)$ & $1(1.54)$ & $55(84.62)$ \\
\hline Experimental group & 65 & $44(67.69)$ & $18(27.69)$ & $3(4.62)$ & $0(0.00)$ & $62(95.38)$ \\
\hline $\mathrm{x}^{2}$ & & 4.433 & 0.350 & 5.082 & 1.552 & 6.432 \\
\hline $\mathrm{P}$ & & 0.035 & 0.554 & 0.024 & 0.213 & 0.011 \\
\hline
\end{tabular}

Note: $\mathrm{x}^{2}$ and $\mathrm{P}$ represent comparisons of Cure Rate and Total Effective Treatment Rate in the Control group and Experimental group.

Table 2. Comparison of Damaged Skin Area and Pigmentation Rate of the Two Groups [n (\%)].

\begin{tabular}{ccccccc}
\hline \multirow{2}{*}{ Group } & \multirow{2}{*}{ Cases } & \multicolumn{5}{c}{ Reduction of Damaged Skin Area } \\
\cline { 3 - 5 } & & $<40 \%$ & $40 \% \sim 60 \%$ & $60 \% \sim 80 \%$ & $>80 \%$ & \multirow{2}{*}{ Pigmentation Rate } \\
\hline Control group & 65 & $4(6.15)$ & $22(33.85)$ & $15(23.08)$ & $25(38.46)$ & $19(29.23)$ \\
Experimental group & 65 & $2(3.08)$ & $8(12.31)$ & $16(24.62)$ & $39(60.00)$ & $8(12.31)$ \\
$\mathrm{X}^{2}$ & & 1.071 & 12.294 & 0.067 & 9.955 & 8.035 \\
$\mathrm{P}$ & & 0.301 & 0.001 & 0.796 & 0.002 & 0.005 \\
\hline
\end{tabular}

Note: $\mathrm{x}^{2}$ and P represent comparisons of Damaged Skin Area and Pigmentation Rate in the Control group and Experimental group.

Table 3. Comparison of Incidence of Adverse Reactions of the Two Groups [n (\%)].

\begin{tabular}{cccccc}
\hline Group & Cases & Dry Desquamation & Pruritus & Rubella & \multicolumn{2}{c}{$\begin{array}{c}\text { Incidence of Adverse } \\
\text { Reactions }\end{array}$} \\
\hline Control group & 65 & $3(4.62)$ & $3(4.62)$ & $2(3.08)$ & $8(12.31)$ \\
Experimental group & 65 & $1(1.54)$ & $0(0.00)$ & $0(0.00)$ & $1(1.54)$ \\
$\mathrm{x}^{2}$ & & 0.782 & 2.337 & 1.542 & 4.333 \\
$\mathrm{P}$ & & 0.377 & 0.126 & 0.214 & 0.037 \\
\hline
\end{tabular}

Note: $\mathrm{x}^{2}$ and P represent comparisons of Incidence of Adverse Reactions in the Control group and Experimental group.
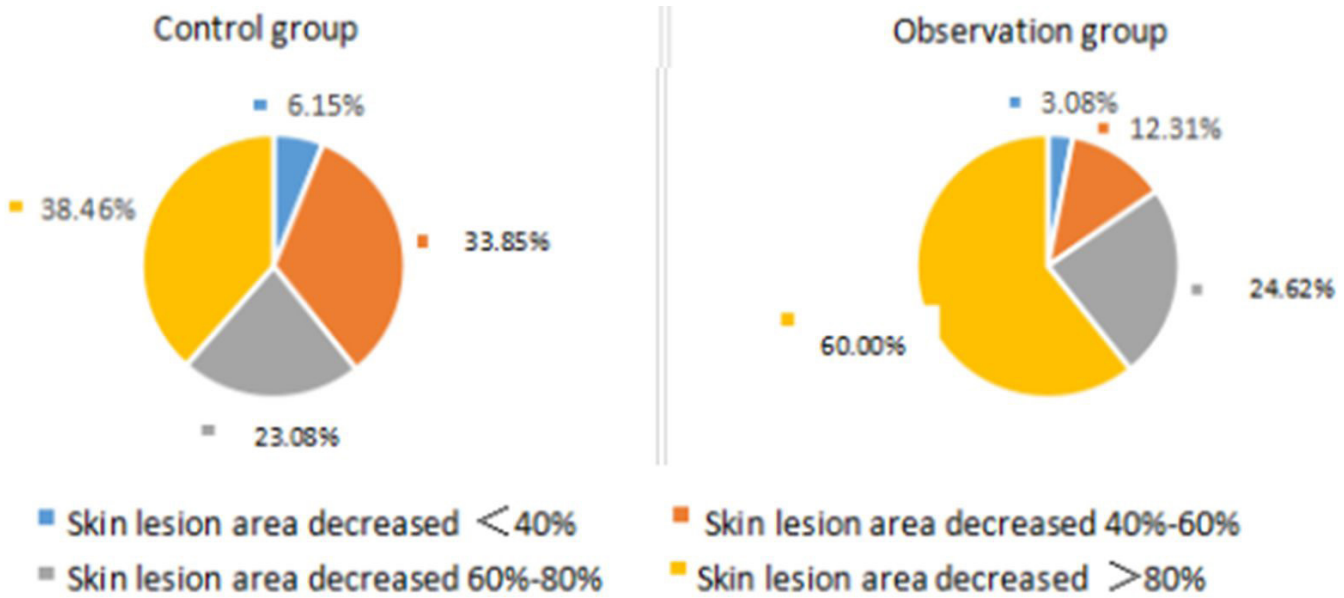

Figure 1. Comparison of Reduction of Damaged Skin Area of the Two Groups.

greenish brown birthmark may be that melanocytes fail to stay in the dermis through the interface layer between the epidermis and dermis during migration from neural crest to epidermis during embryonic development (Dominguez \& Dobke, 2017). The facial greenish brown birthmark usually occurs symmetrically from bilateral zygomatics, and gradually develops and extends to bilateral forehead, bilateral temporal, periocular and alar of nose. It may also occur in bilateral buccal areas, showing round, oval or irregular blue brown and light brown patches (Yu et al., 2018). The pathological analysis showed that a small number of spindle cells with pigment were scattered in the superficial dermis by greenish brown birthmark HE staining. Electron microscopy confirmed that there were small melanosomes in these cells, and HMB45 was positive, which was a birthmark cell (Drzewiecki et al., 2017). The main purpose of clinical treatment of facial greenish brown birthmark is to effectively remove melanosomes in melanocytes without damaging surrounding cells (Sunassee et al., 2017).

In recent years, laser technology has developed and become mature gradually, used widely in clinical disease treatment (Martins et al., 2017). Turning-Q switch technology is a technology that compresses the general output continuous laser energy into 
a pulse with a very narrow width for emission, so as to improve the peak power of the light source by several orders of magnitude (Perron et al., 2018). Turning-Q laser treatment instrument 1064nm compresses the high-energy long pulse laser with wavelength of $1064 \mathrm{~nm}$ to a very narrow width. Based on the principle of selective photothermal effect, the melanosomes in melanocytes are selectively destroyed, which makes the pigment particles absorb extremely high laser energy in a moment, expand and break up. Then the phagocytes of the body digest and dispose the pigment particles by themselves and discharge them out of the body In addition, to achieve the purpose of desalination and removal of color spots (Yang et al., 2017). The pulse width of $100 \mathrm{~ns}$ is less than the target tissue thermal relaxation time, it will not cause damage to the surrounding normal cells, and the treatment is safe, without scars, with non-invasive or minimally invasive effect. Through turning-Q laser treatment for facial greenish brown birthmark, a few patients may have inflammation and edema reaction due to local laser irradiation, or temporary pigmentation caused by forced removal of scab during wound healing. Many studies have confirmed that pigment absorption is a relatively slow biological process, which usually takes several months or even one year to remove pigment particles completely. Therefore, laser treatment of greenish brown birthmark needs to extend the interval time to ensure that pigment can be removed completely and number of laser irradiation can be reduced (Zhang et al., 2017).

In this study, the patients in the control group were treated with conventional cold compress with water bag after laser irradiation to cool down, relieve pain and relieve edema; The experimental group was treated with Chuangfukang collagen mask cold external application immediately after laser treatment. It was found that the cure rate and the total effective rate of the treatment group were significantly improved. The area of greenish brown birthmark was smaller than that in the control group in 1 weeks after treatment. The pigmentation rate was lower than that in the control group, and the incidence of ill reaction was significantly lower in the 6 month after treatment than in the control group. It turns out that turning-Q laser in combination with topical Chuangfukang collagen mask is helpful to improve the curative effect, accelerate the absorption of pigment granules, reduce pigmentation and improve the safety of treatment. Chuangfukang collagen mask is a medical mask. Its main functional ingredient is active collagen solution. It has mild repair of damaged skin barrier, replenish collagen and improve metabolism (Cornelius et al., 2017). In the research, Trivedi and other researchers (Trivedi et al., 2017) pointed out that collagen synthesis can promote skin wound healing, and has an obvious effect on improving its elasticity and firmness, which is conducive to skin whitening, and that collagen plays a significant role in beauty. The application of mask immediately after laser treatment will not only reduce the temperature and analgesic effect effectively, the active collagen and various amino acids will also provide adequate nutrition for the damaged skin, promote the improvement of microcirculation and metabolism of epidermal cells, accelerate the repair and regeneration of cell tissue, and tyrosinase in the colloid protein can inhibit the tyrosinase active center in the skin effectively. Tyrosine is catalysed to form DOPA quinone, thus inhibiting melanin production and preventing pigmentation (Shekhter et al., 2019). After greenish brown birthmark was treated with turning-Q laser treatment instrument $1064 \mathrm{~nm}$., cold external application of Chuangfukang collagen mask for 5 consecutive days, combined with antibiotic ointment, it will improve the facial skin inflammation and edema reaction greatly. Compared with conventional cold pack, the skin's overall restoration effect and inhibition of pigmentation effect are significantly improved.

In conclusion, the curative effect of patients with greenish brown birthmark treated with turning- $Q$ laser in combination with topical Chuangfukang collagen mask can be improved significantly. It will accelerate the absorption of pigment granules, reduce pigmentation, and reduce the incidence of adverse reactions after treatment. Besides, it is of positive significance for promoting facial appearance improvement, and is worthy of clinical promotion.

\section{References}

Chathra, N., \& Mysore, V. (2018). Resurfacing of facial acne scars with a new variable-pulsed Er:YAG laser in fitzpatrick skin types IV and V. Journal of Cutaneous and Aesthetic Surgery, 11(1), 20-25. http:// dx.doi.org/10.4103/JCAS.JCAS_4_18. PMid:29731588.

Cornelius, L. P., Raju, V., \& Lalapet, R. A. (2017). A case of epilepsia partialis continua due to linear nevus syndrome with hemimegalencephaly. Journal of Pediatric Neurosciences, 12(2), 203-205. http://dx.doi. org/10.4103/jpn.JPN_17_16. PMid:28904588.

Dominguez, S., \& Dobke, M. (2017). Correction of rhytides, peau d'orange, and thin dermis of the face by neocollagenesis using novel collagen stimulating slurry protocol. Aesthetic Plastic Surgery, 41(5), 1168-1175. http://dx.doi.org/10.1007/s00266-017-0917-9. PMid:28643008.

Drzewiecki, K. E., Malavade, J. N., Ahmed, I., Lowe, C. J., \& Shreiber, D. I. (2017). A thermoreversible, photocrosslinkable collagen bio-ink for free-form fabrication of scaffolds for regenerative medicine. Technology, 5(4), 185-195. http://dx.doi.org/10.1142/ S2339547817500091. PMid:29541655.

Hui, Q., Chang, P., Guo, B., Zhang, Y., \& Tao, K. (2017). The clinical efficacy of autologous platelet-rich plasma combined with ultra-pulsed fractional $\mathrm{CO}_{2}$ laser therapy for facial rejuvenation. Rejuvenation Research, 20(1), 25-31. http://dx.doi.org/10.1089/rej.2016.1823. PMid:27222038.

Imrigha, N., Bidin, N., Lau, P. S., Musa, N., Zakaria, N., \& Krishnan, G. (2017). Photobiomodulation therapy on wound treatment subsequent to Q-switched Nd: YAG laser tattoo removal in rat model. Journal of Biophotonics, 10(10), 1287-1291. http://dx.doi. org/10.1002/jbio.201600295. PMid:28464516.

Maligieri, L. A. O., Neves, L. M. G., de Morais, D. T., Domingues, R. F., Aro, A. A., Pimentel, E. R., do Amaral, M. E. C., Esquisatto, M. A. M., Santos, G. M. T., \& Mendonça, F. A. S. (2017). Differing energy densities with laser $670 \mathrm{~nm}$ InGaP controls inflammation and collagen reorganization in burns. Burns, 43(7), 1524-1531. http://dx.doi.org/10.1016/j.burns.2017.04.008. PMid:28778761.

Martins, M. F., Kiefer, K., Kanecadan, L., Garcia, P. N., Belfort, R. N., \& Allemann, N. (2017). Comparisons of choroidal nevus measurements obtained using 10 - and $20-\mathrm{MHz}$ ultrasound and spectral domain optical coherence tomography. Arquivos Brasileiros de Oftalmologia, 80(2), 78-83. http://dx.doi.org/10.5935/0004-2749.20170020. PMid:28591278. 
Perron, E., Pissaloux, D., Charon Barra, C., Karanian, M., Lamant, L., Parfait, S., Alberti, L., \& de la Fouchardière, A. (2018). Melanocytic Myxoid Spindle Cell Tumor With ALK Rearrangement (MMySTAR): report of 4 cases of a nevus variant with potential diagnostic challenge. The American Journal of Surgical Pathology, 42(5), 595-603. PMid:29635259.

Shekhter, A. B., Fayzullin, A. L., Vukolova, M. N., Rudenko, T. G., Osipycheva, V. D., \& Litvitsky, P. F. (2019). Medical applications of collagen and collagen-based materials. Current Medicinal Chemistry, 26(3), 506-516. http://dx.doi.org/10.2174/0929867325666171205170339. PMid:29210638.

Simunovic, F., Schlager, S., Montanari, M., \& Iblher, N. (2017). Prospective 3D analysis of facial soft tissue augmentation with calcium hydroxylapatite. Journal of Cosmetic and Laser Therapy, 19(5), 283-289. http://dx.doi.org/10.1080/14764172.2017.130741 1. PMid:28328289.

Sunassee, A., Kerkvliet, A. M., \& Jassim, A. D. (2017). Combined melanocytic nevus, superficial congenital and deep penetrating types with fibroepithelioma of pinkus, collision tumor: a case report. South Dakota Medicine, 70(8), 363-365.

Trivedi, M. K., Gangwar, M., Mondal, S. C., \& Jana, S. (2017). Protective effects of tetrahydrocurcumin (THC) on fibroblast and melanoma cell lines in vitro: it's implication for wound healing. Journal of Food Science and Technology, 54(5), 1137-1145. http://dx.doi.org/10.1007/ s13197-017-2525-8. PMid:28416863.

Yang, L., Wu, H., Zhang, M., Xie, S., Ye, D., \& Tang, S. (2017). Application of amniotic membrane-living skin equivalent in repairing skin defect after removal of congenital giant nevus. Chinese Journal of Reparative and Reconstructive Surgery, 31(12), 1495-1499. PMid:29806394.

Yu, X., Zhang, J., Gu, Y., Deng, D., Wu, Z., Bao, L., Li, M., \& Yao, Z. (2018). CHILD syndrome mimicking verrucous nevus in a Chinese patient responded well to the topical therapy of compound of simvastatin and cholesterol. Journal of the European Academy of Dermatology and Venereology, 32(7), 1209-1213. http://dx.doi. org/10.1111/jdv.14788. PMid:29341259.

Zhang, Q. L., Dong, C. Y., Liu, L., Wen, S. P., \& Wang, X. Y. (2019). Beijing da xue xue bao. Journal of Peking University (Health Sciences), 51(1), 28-34.

Zhang, Q., Su, J., Ye, M. M., Liu, Y. L., Liu, J. Y., Mei, F., \& Gao, F. (2017). Proliferative nodules arising from a giant congenital nevocytic nevus: a case report and review of the literatures. Journal of Clinical Dermatology, 46(8), 557-560. 